\title{
Acoustic scattering in a waveguide with a height discontinuity bridged by a membrane: a tailored Galerkin approach.
}

by

Jane B. Lawrie

Department of Mathematics, Brunel University London

Uxbridge, UB8 3PH, UK

Email: Jane.Lawrie@brunel.ac.uk

and

\author{
Muhammad Afzal \\ Department of Mathematics \\ Capital University of Science and Technology, Kahuta Road \\ Islamabad, Pakistan \\ Email: dr.mafzal@cust.edu.pk
}

\begin{abstract}
This article is concerned with the reflection and transmission of fluid-structure coupled waves at the junction between two flexible waveguides of different heights. Unlike previous studies, in which the waveguides are joined at the height discontinuity by a rigid or soft strip, here the height discontinuity is bridged by a membrane. The aims are first to develop a solution method that enables a wide range of conditions to be applied at the edges of the bridging membrane, and then to ascertain the effect that these have on the reflected and transmitted fields. Numerical results are presented which confirm that that the conditions applied at the edges of the bridging membrane do have a significant effect on the reflected and transmitted components of power.
\end{abstract}

Keywords: Acoustic scattering; fluid-structure coupled waves; mode-matching; Galerkin procedure; flexible walls; thin plates and membranes. 


\section{Introduction}

Mode-matching methods provide a convenient and powerful means by which to address a wide range of problems involving scattering in ducts or channels. The approach was originally developed for the solution of canonical problems in which the underlying eigen-systems were of Sturm-Liouville type. In recent years, however, the method has been advanced and extended in many directions. In particular, multimodal and hybrid methods have been developed to deal with rigid walled ducts with non-canonical geometries or lined inclusions [1]-[6], and generalised orthogonality relations (ORs) have been derived for problems involving propagation in ducts/channels with high order boundary conditions [7]-[9]. The methods associated with the latter class of ORs have been extended to three dimensional problems with rectangular cross-sections [10]-[12], and the ability of these methods to handle singularities in the fluid velocity field has been studied [13]. These developments have enabled a much wider range of problems in acoustics and hydrodynamics [14]-[16] to be addressed using mode-matching and related methods.

This article is concerned with the reflection and transmission of fluid-structure coupled waves at the junction between two flexible waveguides of different heights. Forcing is introduced in the form of a wave incident towards the junction; both the fundamental mode and the first higher mode are considered in this role. Unlike previous studies [8], [13], in which the waveguides are joined at the height discontinuity by a rigid or soft strip, here the strip joining the waveguides is also flexible. This type of junction can be found in heating, ventilation and air conditioning (HVAC) systems and also vehicle exhaust systems. In both situations the walls of the expansion chamber which forms part of a silencer are not rigid. In most practical applications the flexible surfaces would be modelled using, for example, the thin plate equation or Mindlin theory, however, in this article a prototype problem is considered in which all flexible surfaces are modelled as membranes.

The model problem can be solved using mode-matching in conjunction with the well established Galerkin procedure - a technique that has been extensively studied 
and used to solve a wide range of related problems [17]-[22]. Fundamental to the Galerkin procedure is the assumption that a particular characteristic of the physical problem can be modelled as a expansion in terms of appropriately chosen basis functions. The basis functions may be chosen, for example, to model a corner singularity in the fluid velocity field [17], [22] or, as herein, to ensure that the conditions at the edges of a finite membrane or plate are satisfied [21]-[20]. Thus, in general, a different set of basis functions is required for each variation of a given physical problem and, in the current context, this can lead to a further "layer" of root-finding.

The aims of this article are: first, to develop a solution method that enables a wide range of conditions to be applied at the the edges of the vertical membrane without the need to change basis functions; second, to ascertain the effect that the bridging membrane has on the reflected and transmitted fields. The new approach is a variation of the Galerkin procedure. The displacement of the vertical membrane is represented as a modal expansion using the eigenfunctions for the duct section of greater height. Key to the success of this approach are the properties of these eigenfunctions, namely, their linearly dependence and the Green's function representation [9]. The Green's function is used to obtain a closed-form expression for the vertical membrane displacement in terms of the the fluid modal amplitudes and this expression has sufficient degrees of freedom to enable a wide range of edge conditions to be applied.

The article is organised as follows. In section 2, the two-dimensional (2D) model problem is described and the corresponding boundary value problem is stated. The mode-matching solution is executed in sections 3 and 4 . First the problem studied by Warren et al [8] is revisited and then the current problem is solved using the two methods discussed above. A study of the reflected and transmitted components of power for three different sets of edge conditions and two different incident waves is presented in section 5. The tailored Galerkin approach is then validated by demonstrating the conditions at matching interface are fully satisfied. The relative merits of the two solution methods are discussed in section 6 . 


\section{The model problem}

Consider a two-dimensional waveguide comprising two semi-infinite sections of different heights $\bar{a}$ and $\bar{b}, \bar{b}>\bar{a}$, that meet along a vertical interface which, in a Cartesian frame of reference, is specified by $\bar{x}=0$. The lower wall of the waveguide lies along $\bar{y}=0,-\infty<\bar{x}<\infty$ and is acoustically hard whilst the upper boundaries comprise membranes which lie on $\bar{y}=\bar{a},-\infty<\bar{x} \leq 0$ and $\bar{y}=\bar{b}, 0 \leq \bar{x}<\infty$ respectively. At $\bar{x}=0$, the two duct sections are joined by means of a vertical strip occupying $\bar{a} \leq \bar{y} \leq \bar{b}$. Two cases are considered: a rigid strip and a membrane strip, see figure 1. A compressible fluid of density $\rho$ and sound speed $c$ occupies the interior region of the duct.

An incident wave of harmonic time dependence $e^{-i \omega \bar{t}}$, where $\omega=c k$ is the radian frequency in which $k$ is the fluid wavenumber, propagates in the positive $\bar{x}$ direction towards $\bar{x}=0$. On non-dimensionalising the boundary value problem according to $k \bar{x}=x, k \bar{y}=y$ and $\omega \bar{t}=t$, the governing equation is:

$$
\left(\nabla^{2}+1\right) \phi=0
$$

where $\phi(x, y)$ is the non-dimensional reduced (time independent) velocity potential which may be expressed as

$$
\phi(x, y)=\left\{\begin{array}{ll}
\phi_{1}(x, y), & x<0,0 \leq y \leq a \\
\phi_{2}(x, y), & x>0,0 \leq y \leq b
\end{array} .\right.
$$

The boundary condition at the rigid base is

$$
\frac{\partial \phi_{j}}{\partial y}=0, \quad y=0, \quad-\infty<x<\infty, j=1,2
$$

For the upper boundaries of the waveguide the non-dimensional membrane boundary condition is

$$
\left(\frac{\partial^{2}}{\partial x^{2}}+\mu^{2}\right) \phi_{j y}+\alpha \phi_{j}=0, \quad j=1,2
$$

where for $j=1$ the condition is applied at $y=a, x<0$ and for $j=2$ it is applied at $y=b, x>0$ respectively. (The subscript $y$ here and henceforth indicates differentiation with respect to this variable.) The non-dimensional parameter $\mu=$ 
$c / c_{m}$ is the in vacuo membrane wavenumber and $\alpha=\omega^{2} \rho /\left(T k^{3}\right)$ is a fluid loading parameter. Here $T$ denotes the membrane tension and $c_{m}=\sqrt{T / \rho_{m}}$ is the in vacuo speed of waves on the membrane in which $\rho_{m}$ is the membrane mass per unit area.

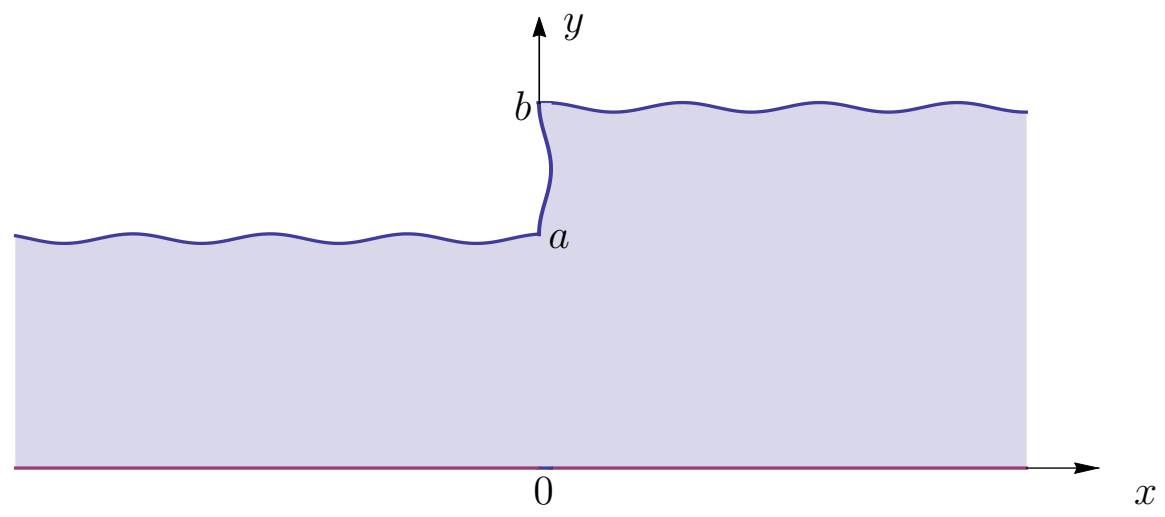

Figure 1: The duct geometry (depicting the membrane strip) in which $a=k \bar{a}$ and $b=k \bar{b}$.

The velocity potentials can be expressed as eigenfunction expansions of the form:

$$
\phi_{1}(x, y)=F_{\ell} \cosh \left(\tau_{\ell} y\right) e^{i \eta_{\ell} x}+\sum_{n=0}^{\infty} A_{n} \cosh \left(\tau_{n} y\right) e^{-i \eta_{n} x}, \quad x<0
$$

and

$$
\phi_{2}(x, y)=\sum_{n=0}^{\infty} B_{n} \cosh \left(\gamma_{n} y\right) e^{i s_{n} x}, \quad x>0
$$

where the coefficients $A_{n}$ and $B_{n}$ are the complex amplitudes of $n^{\text {th }}$ reflected and transmitted modes respectively. The first term in (5) is an incident wave having amplitude $F_{\ell}=\sqrt{\alpha /\left(C_{\ell} \eta_{\ell}\right)}$, which is chosen to ensure unit incident power $\left(C_{\ell}\right.$ is defined in the text just below equation (10)). The counter $\ell$ is taken to be either 0 or 1 corresponding respectively to the fundamental (structural) mode or the first higher mode. The non-dimensional wave numbers, $\eta_{n}=\sqrt{\tau_{n}^{2}+1}$ and $s_{n}=\sqrt{\gamma_{n}^{2}+1}$, are either positive real or have positive imaginary part. The quantities $\tau_{n}$ and $\gamma_{n}$, $n=0,1,2, \ldots$ are the roots of the dispersion relations $K(\tau)=0$ and $L(\gamma)=0$ respectively where

$$
K(\tau)=\left(\tau^{2}+1-\mu^{2}\right) \tau \sinh (\tau a)-\alpha \cosh (\tau a),
$$


and

$$
L(\gamma)=\left(\gamma^{2}+1-\mu^{2}\right) \gamma \sinh (\gamma b)-\alpha \cosh (\gamma b)
$$

These roots are found numerically and their properties are discussed in, for example, [13].

The eigenfunctions satisfy a generalised orthogonality relation (OR) which, for those relevant to the right hand duct (that lying in $x>0$ ), is

$$
\alpha \int_{0}^{b} \cosh \left(\gamma_{m} y\right) \cosh \left(\gamma_{n} y\right) d y=\delta_{m n} D_{m}-\gamma_{m} \gamma_{n} \sinh \left(\gamma_{m} b\right) \sinh \left(\gamma_{n} b\right)
$$

where $\delta_{m n}$ is the Kronecker delta and

$$
D_{m}=\frac{\alpha b}{2}+\left(\frac{3 \gamma_{m}^{2}+1-\mu^{2}}{2 \gamma_{m}^{2}}\right)\left[\gamma_{m} \sinh \left(\gamma_{m} b\right)\right]^{2}
$$

The OR for the duct lying in $x<0$ is identical in structure and can be obtained from (9)-(10) on replacing $b$ with $a, \gamma_{m}$ with $\tau_{m}$ and $D_{m}$ with $C_{m}$. In addition to the orthogonality property (9), the eigenfunctions are linearly dependent and a Green's function can be constructed. In terms of the eigenfunctions for the duct lying in $x>0$, the Green's function takes the form:

$$
\begin{array}{r}
\alpha \sum_{q=0}^{\infty} \frac{\cosh \left(\gamma_{q} v\right) \cosh \left(\gamma_{q} y\right)}{D_{q}}=\delta(y-v)+\delta(y+v)+\delta(y+v-2 b) \\
0 \leq y, v \leq b
\end{array}
$$

where $\delta(y)$ is the usual Dirac delta function and linear dependence is indicated by:

$$
\mathcal{Z}(y)=\sum_{q=0}^{\infty} \frac{\gamma_{q} \sinh \left(\gamma_{q} b\right) \cosh \left(\gamma_{q} y\right)}{D_{q}}=0, \quad 0 \leq y \leq b
$$

It is important to note that although $\mathcal{Z}(y)=0,0 \leq y \leq b$, its derivatives are non-zero. Of particular note are the results $\mathcal{Z}^{\prime}(b)=1$ and

$$
\mathcal{Z}^{\prime \prime}(y)=\left(\mu^{2}-1\right) \mathcal{Z}(y)+2 \delta(y-b), \quad 0 \leq y \leq b,
$$

the latter being obtained by using (8).

The eigenfunction expansion (5) comprises an incident wave and a reflected field whereas (6) is the transmitted field. The non-dimensional reflected and transmitted 
powers are expressed in terms of the modal coefficients as:

$$
\mathcal{E}_{1}=\frac{1}{\alpha} \sum_{m=0}^{J_{1}-1}\left|A_{m}\right|^{2} C_{m} \eta_{m} \quad \text { and } \quad \mathcal{E}_{2}=\frac{1}{\alpha} \sum_{m=0}^{J_{2}-1}\left|B_{m}\right|^{2} D_{m} s_{m},
$$

where $J_{1}\left(J_{2}\right)$ is the number of cut-on modes in the left(right) hand duct. These expressions incorporate both the fluid and the structure-borne components of the reflected and transmitted powers [8], [13]. As mentioned above, the choice of $F_{\ell}$ is such that the incident power is unity and it follows that

$$
\mathcal{E}_{1}+\mathcal{E}_{2}=1
$$

\subsection{The mode-matching solution}

At the matching interface $(x=0)$ the fluid pressure is continuous, thus

$$
\phi_{1}=\phi_{2}, \quad 0 \leq y \leq a
$$

On substituting the eigenfunction expansions (5) and (6) into (16), multiplying by $\alpha \cosh \left(\tau_{m} y\right)$ and using the OR (equivalent to $(9)$ ) for the duct lying in $x<0$, it is found that

$$
A_{m}=-F_{\ell} \delta_{m \ell}+E_{1} \frac{\tau_{m} \sinh \left(\tau_{m} a\right)}{C_{m}}+\frac{\alpha}{C_{m}} \sum_{n=0}^{\infty} B_{n} R_{m n},
$$

where $E_{1}=\phi_{1 y}(0, a)$ and

$$
R_{m n}=\int_{0}^{a} \cosh \left(\tau_{m} y\right) \cosh \left(\gamma_{n} y\right) d y
$$

Note that the roots of dispersion relation defined in (7) and (8) are either real or imaginary which ensures that $R_{m n}$ is always real.

In addition to continuity of pressure, a second condition giving appropriate information about the normal component of the fluid velocity is required. Further, edge conditions must be applied at the points where the membranes are either connected to the rigid strip or, in the case of the bridging membrane, to each other. 


\section{$3 \quad$ Rigid strip}

Before considering the membrane strip it is worthwhile revisiting the problem studied by Warren et al [8] in which the vertical strip is rigid. The appropriate velocity condition at $x=0$ is

$$
\frac{\partial \phi_{2}}{\partial x}=\left\{\begin{array}{cc}
\frac{\partial \phi_{1}}{\partial x}, & 0 \leq y<a \\
0, & a \leq y \leq b
\end{array}\right.
$$

On substituting (5) and (6) into (19), multiplying by $\alpha \cosh \left(\gamma_{m} y\right)$ and using (9), it is found that

$$
B_{m}=\frac{\alpha F_{\ell} \eta_{\ell} R_{\ell m}}{D_{m} s_{m}}-i E_{2} \frac{\gamma_{m} \sinh \left(\gamma_{m} b\right)}{D_{m} s_{m}}-\frac{\alpha}{D_{m} s_{m}} \sum_{n=0}^{\infty} A_{n} \eta_{n} R_{n m}
$$

Then, on using (17) to eliminate $A_{n}$ from (20):

$$
\begin{aligned}
B_{m} & =\frac{2 \alpha F_{\ell} \eta_{\ell} R_{\ell m}}{D_{m} s_{m}}-\frac{\alpha E_{1}}{D_{m} s_{m}} \sum_{n=0}^{\infty} \frac{\eta_{n} \tau_{n} \sinh \left(\tau_{n} a\right) R_{n m}}{C_{n}} \\
& -i E_{2} \frac{\gamma_{m} \sinh \left(\gamma_{m} b\right)}{D_{m} s_{m}}-\frac{\alpha^{2}}{D_{m} s_{m}} \sum_{n=0}^{\infty} B_{n} \sum_{q=0}^{\infty} \frac{\eta_{q} R_{q n} R_{q m}}{C_{q}} .
\end{aligned}
$$

The values of the constants $E_{1}$ and $E_{2}$ depend on the choice of the edge conditions imposed on the two semi-infinite membranes. Here and henceforth it is assumed that both have zero displacement at $x=0, y=a, b$. That is

$$
\phi_{1 y}(0, a)=\phi_{2 y}(0, b)=0 \text {. }
$$

On applying conditions (22), it is found that $E_{1}=0$ and

$$
\begin{aligned}
E_{2} S_{1} & =-i \alpha \sum_{m=0}^{\infty} \frac{\gamma_{m} \sinh \left(\gamma_{m} b\right)}{D_{m} s_{m}}\left\{2 F_{\ell} \eta_{\ell} R_{\ell m}-E_{1} \sum_{n=0}^{\infty} \frac{\eta_{n} \tau_{n} \sinh \left(\tau_{n} a\right) R_{n m}}{C_{n}}\right\} \\
& +i \alpha^{2} \sum_{m=0}^{\infty} \frac{\gamma_{m} \sinh \left(\gamma_{m} b\right)}{D_{m} s_{m}} \sum_{n=0}^{\infty} B_{n} \sum_{q=0}^{\infty} \frac{\eta_{q} R_{q n} R_{q m}}{C_{q}}
\end{aligned}
$$

where

$$
S_{1}=\sum_{m=0}^{\infty} \frac{\left[\gamma_{m} \sinh \left(\gamma_{m} b\right)\right]^{2}}{D_{m} s_{m}}
$$

Equations (21) and (23) can be truncated and solved numerically. 


\section{Membrane strip}

In this section the rigid vertical strip is replaced by a membrane. Thus, the nondimensional velocity condition (19) is replaced by

$$
\frac{\partial \phi_{2}}{\partial x}=\left\{\begin{array}{cc}
\frac{\partial \phi_{1}}{\partial x}, & 0 \leq y<a \\
w(y), & a<y<b
\end{array},\right.
$$

where the (non-dimensional) displacement of the vertical membrane, $w(y)$, satisfies

$$
w_{y y}+\mu^{2} w-\alpha \phi_{2}=0, \quad a<y<b .
$$

In addition to (22), edge conditions must now be applied at either end of the vertical membrane. Three different sets of edge conditions are considered. It is convenient to assume that the displacement is zero at $y=a$ but that at $y=b$ the membrane displacement, gradient or a linear combination of the two is assumed to be zero. These are discussed in more detail later in the text. Two solution methods are considered: the first is the well known Galerkin approach; the second is a tailored version of the latter which exploits the properties of the eigenfunctions for the duct lying in $x>0$.

\subsection{Solution using the Galerkin procedure}

Fundamental to the Galerkin approach is the selection of an appropriate set of mutually orthogonal basis functions to describe the displacement of the vertical membrane. An appropriate set is one in which each function satisfies the edge conditions imposed at $y=a, b$. Given that $w(a)=0, w(y)$ can be expressed as a Fourier sine series of the form

$$
w(y)=\sum_{n=n_{0}}^{\infty} G_{n} \sin \left[\lambda_{n}(y-a)\right],
$$

where the Fourier coefficients $G_{n}, n=1,2,3, \ldots$ are unknown. Each term of the series satisfies the zero displacement condition at $y=a$ and the eigenvalues $\lambda_{n}$ are chosen to satisfy the remaining condition at $y=b$. It follows that $n_{0}$ is either 0 or 1 depending on the choice of $\lambda_{n}$. The coefficients $G_{n}$ can be expressed in terms of the fluid modal amplitudes $B_{n}, n=0,1,2, \ldots$ by using (26). 
On substituting (6) and (27) into (26) it is found that

$$
\sum_{n=0}^{\infty} G_{n}\left\{\mu^{2}-\lambda_{n}^{2}\right\} \sin \left[\lambda_{n}(y-a)\right]=\alpha \sum_{n=0}^{\infty} B_{n} \cosh \left(\gamma_{n} y\right), \quad a \leq y \leq b .
$$

Then, on multiplying $(28)$ by $\sin \left[\lambda_{m}(y-a)\right]$ and using the usual OR:

$$
G_{m}=\frac{\alpha}{\Delta_{m}\left\{\mu^{2}-\lambda_{m}^{2}\right\}} \sum_{n=0}^{\infty} B_{n} P_{m n}
$$

where

$$
P_{m n}=\int_{a}^{b} \sin \left[\lambda_{m}(y-a)\right] \cosh \left(\gamma_{n} y\right) d y
$$

and

$$
\Delta_{m}=\frac{b-a}{2}-\frac{\sin \left[2 \lambda_{m}(b-a)\right]}{4 \lambda_{m}} .
$$

Having obtained a Fourier sine series representation for $w(y)$ this can be substituted, together with (5) and (6), into the velocity condition (25). On multiplying through by $\alpha \cosh \left(\gamma_{m} y\right)$ and using (9), it is found that

$$
\begin{aligned}
B_{m} & =\frac{\alpha F_{\ell} \eta_{\ell} R_{\ell m}}{D_{m} s_{m}}-\frac{\alpha}{D_{m} s_{m}} \sum_{n=0}^{\infty} A_{n} \eta_{n} R_{n m}-i E_{2} \frac{\gamma_{m} \sinh \left(\gamma_{m} b\right)}{D_{m} s_{m}} \\
& -\frac{i \alpha^{2}}{D_{m} s_{m}} \sum_{n=0}^{\infty} B_{n} \sum_{q=0}^{\infty} \frac{P_{q m} P_{q n}}{\Delta_{q}\left\{\mu^{2}-\lambda_{q}^{2}\right\}} .
\end{aligned}
$$

On using (17) to eliminate $A_{n}$ from (32):

$$
\begin{aligned}
B_{m} & =\frac{2 \alpha F_{\ell} \eta_{\ell} R_{\ell m}}{D_{m} s_{m}}-\frac{\alpha E_{1}}{D_{m} s_{m}} \sum_{n=0}^{\infty} \frac{\eta_{n} \tau_{n} \sinh \left(\tau_{n} a\right) R_{n m}}{C_{n}}-i E_{2} \frac{\gamma_{m} \sinh \left(\gamma_{m} b\right)}{D_{m} s_{m}} \\
& -\frac{\alpha^{2}}{D_{m} s_{m}} \sum_{n=0}^{\infty} B_{n} \sum_{q=0}^{\infty}\left\{\frac{\eta_{q} R_{q n} R_{q m}}{C_{q}}+\frac{i P_{q m} P_{q n}}{\Delta_{q}\left\{\mu^{2}-\lambda_{q}^{2}\right\}}\right\} .
\end{aligned}
$$

Before this system of equations can be truncated and solved the edge conditions (22) must be imposed and the eigenvalues $\lambda_{n}$ must be specified. Equations (22) imply that $E_{1}=0$; the corresponding value of $E_{2}$ is (as in section 3 ) obtained by multiplying (33) by $\gamma_{m} \sinh \left(\gamma_{m} b\right)$ and summing over $m$. Thus,

$$
\begin{aligned}
E_{2} S_{1} & =-i \alpha \sum_{m=0}^{\infty} \frac{\gamma_{m} \sinh \left(\gamma_{m} b\right)}{D_{m} s_{m}}\left\{2 F_{\ell} \eta_{\ell} R_{\ell m}-E_{1} \sum_{n=0}^{\infty} \frac{\eta_{n} \tau_{n} \sinh \left(\tau_{n} a\right) R_{n m}}{C_{n}}\right\} \\
& +i \alpha^{2} \sum_{m=0}^{\infty} \frac{\gamma_{m} \sinh \left(\gamma_{m} b\right)}{D_{m} s_{m}} \sum_{n=0}^{\infty} B_{n} \sum_{q=0}^{\infty}\left\{\frac{\eta_{q} R_{q n} R_{q m}}{C_{q}}+\frac{i P_{q m} P_{q n}}{\Delta_{q}\left\{\mu^{2}-\lambda_{q}^{2}\right\}}\right\}
\end{aligned}
$$


The choice of eigenvalues, $\lambda_{n}$, is dictated by the behaviour of the bridging membrane at $y=b$.

i) Zero displacement at $y=a$ and $y=b$

This set of edge conditions is the most physically realistic. Clearly if the displacement is zero at $y=b$ then $\sin \left[\lambda_{n}(b-a)\right]=0$, so:

$$
\lambda_{n}=\frac{n \pi}{b-a}, \quad n=1,2,3, \ldots
$$

ii) Zero displacement at $y=a$; zero gradient at $y=b$

A zero gradient edge condition corresponds to the idealised situation in which the membrane is attached to a light ring which can move freely on a horizontal rod located, for the problem in hand, at $y=b$. For this set of conditions, the correct choice is given by $\cos \left[\lambda_{n}(b-a)\right]=0$, so:

$$
\lambda_{n}=\frac{(n+1 / 2) \pi}{b-a}, \quad n=0,1,2, \ldots
$$

iii) Zero displacement at $y=a$; impedance at $y=b$

The impedance or "spring-like" condition is defined as $w_{y}(b)+\xi w(b)=0$. This condition corresponds to the situation, that in which neither the membrane displacement or its gradient are zero but are coupled. Although an idealised condition, it could be achieved by attaching the membrane to a spring which moves horizontally in the plane $y=b$ and is included to demonstrate the versatility of the solution method. For this set of edge conditions the appropriate values of $\lambda_{n}, n=0,1,2, \ldots$ cannot be expressed explicitly. It transpires that they are the roots of

$$
\lambda \cos [\lambda(b-a)]+\xi \sin [\lambda(b-a)]=0
$$

where $\xi=\bar{\xi} / k$ and $\bar{\xi}$ is the "coupling constant".

Equations (33) and (34) can now be truncated and solved numerically.

As previously mentioned, the basis functions for the Galerkin method are chosen so that the edge conditions are satisfied at either end of the membrane. Thus, each 
set of edge conditions requires a different set of basis functions and for some sets, as in case iii), the eigenvalues must be found numerically. Further, it is not easy to see how conditions such as continuity of gradient (or indeed higher derivatives of the displacement) could be applied at the membrane edges. Whilst such conditions are not relevant to the current study, they are important at the junction of two thin plates, see for example, [12], [13], [23], [24]. In the next section a variation of the Galerkin approach is presented which addresses these issues.

\subsection{Solution using the "tailored" approach}

In order to broaden the range of edge conditions that can be addressed and avoid the need for additional root-finding, it is necessary to express the vertical membrane displacement in terms of a set of basis functions which are already known, and which are non-zero and have non-zero derivatives at $y=a, b$. Thus, it is convenient to express the displacement as a modal expansion using the eigenfunctions for the duct of height $b$. As previously mentioned, key to the success of this approach are the properties of these eigenfunctions, namely, their linearly dependence and the Green's function representation [9]. The membrane displacement is expressed as

$$
w(y)=\sum_{n=0}^{\infty} G_{n} \cosh \left(\gamma_{n} y\right), \quad a \leq y \leq b,
$$

where the modal coefficients $G_{n}$ are unknown (and unrelated to those of section 4.1). On substituting (6) and (38) into (26), it is found that

$$
\sum_{n=0}^{\infty} G_{n}\left\{\gamma_{n}^{2}+\mu^{2}\right\} \cosh \left(\gamma_{n} y\right)=\alpha \sum_{n=0}^{\infty} B_{n} \cosh \left(\gamma_{n} y\right)
$$

Then, on multiplying (39) by $\cosh \left(\gamma_{q} y\right)$ and integrating over $a \leq y \leq b$, it is found that

$$
\sum_{n=0}^{\infty} G_{n}\left\{\gamma_{n}^{2}+\mu^{2}\right\} T_{n q}=\alpha \sum_{n=0}^{\infty} B_{n} T_{n q}
$$

where

$$
T_{n q}=\int_{a}^{b} \cosh \left(\gamma_{q} y\right) \cosh \left(\gamma_{n} y\right) d y
$$


On integrating by parts twice, expression (41) can be rearranged as:

$$
\begin{aligned}
\gamma_{n}^{2} T_{n q}=\gamma_{q}^{2} T_{n q} & +\gamma_{n} \sinh \left(\gamma_{n} b\right) \cosh \left(\gamma_{q} b\right)-\gamma_{n} \sinh \left(\gamma_{n} a\right) \cosh \left(\gamma_{q} a\right) \\
& -\gamma_{q} \sinh \left(\gamma_{q} b\right) \cosh \left(\gamma_{n} b\right)+\gamma_{q} \sinh \left(\gamma_{q} a\right) \cosh \left(\gamma_{n} a\right)
\end{aligned}
$$

This enables (40) to be expressed as:

$$
\begin{aligned}
\sum_{n=0}^{\infty} G_{n} T_{n q}= & -\frac{E_{3} \cosh \left(\gamma_{q} a\right)}{\gamma_{q}^{2}+\mu^{2}}-\frac{E_{4} \cosh \left(\gamma_{q} b\right)}{\gamma_{q}^{2}+\mu^{2}}-\frac{E_{5} \gamma_{q} \sinh \left(\gamma_{q} a\right)}{\gamma_{q}^{2}+\mu^{2}} \\
& -\frac{E_{6} \gamma_{q} \sinh \left(\gamma_{q} b\right)}{\gamma_{q}^{2}+\mu^{2}}+\alpha \sum_{n=0}^{\infty} \frac{B_{n} T_{n q}}{\gamma_{q}^{2}+\mu^{2}}
\end{aligned}
$$

in which the quantities $E_{3}-E_{6}$ are constants. (In fact, $\left.E_{3}=-w_{y}(a), E_{4}=w_{(} b\right)$, $E_{5}=w(a)$ and $E_{6}=-w(b)$ where $w(y)$ is given by (38).) On multiplying (43) by $\alpha \cosh \left(\gamma_{q} y\right) / D_{q}$ and summing over $q$, it is found that:

$$
\begin{array}{r}
\alpha \sum_{n=0}^{\infty} G_{n} \sum_{q=0}^{\infty} \frac{\cosh \left(\gamma_{q} y\right)}{D_{q}} T_{n q}=-E_{3} \psi^{(1)}(y)-E_{4} \psi^{(2)}(y)-E_{5} \psi^{(3)}(y) \\
\quad-E_{6} \psi^{(4)}(y)+\alpha^{2} \sum_{n=0}^{\infty} \sum_{q=0}^{\infty} \frac{B_{n} \cosh \left(\gamma_{q} y\right) T_{n q}}{\left(\gamma_{q}^{2}+\mu^{2}\right) D_{q}}, \quad a \leq y \leq b
\end{array}
$$

where the functions $\psi^{(j)}(y), j=1, \ldots, 4$ are defined by

$$
\begin{array}{r}
\psi^{(1)}(y)=\alpha \sum_{q=0}^{\infty} \frac{\cosh \left(\gamma_{q} a\right) \cosh \left(\gamma_{q} y\right)}{\left(\gamma_{q}^{2}+\mu^{2}\right) D_{q}}, \\
\psi^{(2)}(y)=\alpha \sum_{q=0}^{\infty} \frac{\cosh \left(\gamma_{q} b\right) \cosh \left(\gamma_{q} y\right)}{\left(\gamma_{q}^{2}+\mu^{2}\right) D_{q}}, \\
\psi^{(3)}(y)=\alpha \sum_{q=0}^{\infty} \frac{\gamma_{q} \sinh \left(\gamma_{q} a\right) \cosh \left(\gamma_{q} y\right)}{\left(\gamma_{q}^{2}+\mu^{2}\right) D_{q}}, \\
\psi^{(4)}(y)=\alpha \sum_{q=0}^{\infty} \frac{\gamma_{q} \sinh \left(\gamma_{q} b\right) \cosh \left(\gamma_{q} y\right)}{\left(\gamma_{q}^{2}+\mu^{2}\right) D_{q}} .
\end{array}
$$

The reader is reminded that the aim is to construct $w(y)$ from (44), and also that no explicit OR exists for the functions $\cosh \left(\gamma_{n} y\right)$ on the range $a \leq y \leq b$. The quantity $T_{n q}$ is, however, defined in (41) as an integral and this enables the Green's function (11) to be used in lieu. Thus, on interchanging the orders of summation and 
integration on the left hand side of (44) and using (11), it is found that:

$$
\begin{aligned}
w(y) & =-E_{3} \psi^{(1)}(y)-E_{4} \psi^{(2)}(y)-E_{5} \psi^{(3)}(y)-E_{6} \psi^{(4)}(y) \\
& +\alpha^{2} \sum_{n=0}^{\infty} \sum_{q=0}^{\infty} \frac{B_{n} \cosh \left(\gamma_{q} y\right) T_{n q}}{\left(\gamma_{q}^{2}+\mu^{2}\right) D_{q}}, \quad a \leq y \leq b .
\end{aligned}
$$

Note that the steps taken in obtaining (49) from (40) have involved interchanging the orders of summation and/or integration. These steps are justified only if each summand is suitably convergent - a point which is now addressed. First, however, the reader is requested to note that i) by using (8) it may be shown that $\psi^{(2)}(y)=$ $\left(1-2 \mu^{2}\right) \psi^{(4)}(y) / \alpha+\mathcal{Z}(y)$ where $\mathcal{Z}(y)$ is defined in (12) and ii) any arbitrary constant times $\mathcal{Z}(y)$ can be added to (49). Thus, $w(y)$ can be expressed as

$$
\begin{aligned}
w(y) & =-E_{3} \psi^{(1)}(y)-\hat{E}_{4} \mathcal{Z}(y)-E_{5} \psi^{(3)}(y)-\hat{E}_{6} \psi^{(4)}(y) \\
& +\alpha^{2} \sum_{n=0}^{\infty} \sum_{q=0}^{\infty} \frac{B_{n} \cosh \left(\gamma_{q} y\right) T_{n q}}{\left(\gamma_{q}^{2}+\mu^{2}\right) D_{q}}, \quad a \leq y \leq b .
\end{aligned}
$$

where $\hat{E}_{6}=E_{6}+\left(1-2 \mu^{2}\right) E_{4} / \alpha$ and $\hat{E}_{4}$ is truly arbitrary. On differentiating (44) twice, it is found that

$$
\begin{gathered}
w_{y y}+\mu^{2} w-\alpha \sum_{n=0}^{\infty} B_{n} \cosh \left(\gamma_{n} y\right)=-E_{3} \delta(y-a)-2 \hat{E}_{4} \delta(y-b) \\
-E_{5} \delta^{\prime}(y-a)-\left\{\alpha \hat{E}_{6}+\left(\mu^{2}-1\right) \hat{E}_{4}\right\} \mathcal{Z}(y),
\end{gathered}
$$

which is consistent with (26). Since $\mathcal{Z}(y)=0,0 \leq y \leq b$, expression (51) contains three arbitrary constants. Two of these can be specified by considering the behaviour of the fluid velocity field and the membrane displacement close to the corners.

The velocity potential in the vicinity of the corner at $x=0, y=a$ is determined by considering the local problem. That is, the boundary value problem obtained after converting to polar coordinates $(r, \theta)$, with $r=\sqrt{x^{2}+(y-a)^{2}}$, and re-scaling so as to focus on the region $r<<1$. Then, at leading order, the governing equation reduces to Laplace's equation and the membrane boundary conditions, (4) and (26), simplify significantly. It is found that:

$$
\begin{aligned}
\phi(r, \theta) & \sim \alpha_{0}+\alpha_{1} r \cos \left[\theta+\xi_{1}\right]+\alpha_{2} r^{2} \cos \left[2\left(\theta+\xi_{2}\right)\right]+\alpha_{3} r^{2 / 3} \cos \left[\frac{2}{3}\left(\theta-\frac{\pi}{2}\right)\right] \\
& +\alpha_{4} r^{4 / 3} \cos \left[\frac{4}{3}\left(\theta-\frac{\pi}{2}\right)\right]+\ldots, \quad r \rightarrow 0
\end{aligned}
$$


where $\xi_{1}, \xi_{2}, \alpha_{0}-\alpha_{4}$ are arbitrary constants (the latter not to be confused with the fluid loading parameter $\alpha$ ). It is interesting to note that expression (52) differs from the equivalent expression for a rigid corner through the presence of two extra terms: those pre-multiplied by $\alpha_{1}$ and $\alpha_{2}$. These occur due to the second order derivatives in conditions (4) and (26). Differentiation of (52) reveals that, as $r=\sqrt{x^{2}+(y-a)^{2}} \rightarrow$ 0 , the velocity field has a singularity of $O\left(r^{-1 / 3}\right)$. This is reflected in (51) through the presence of the delta function $\delta(y-a)$. Expression (51) also contains the term $\delta^{\prime}(y-a)$ which, were it to be retained, would generate a Heaviside type of singularity in the membrane displacement. Such behaviour is inconsistent with $w(a)=0$ and is avoided by choosing $E_{5}=0$; a choice which also ensures that the preceding steps involving the interchanging of the orders of summation and summation/integration are justified.

In contrast, the velocity potential in the vicinity of the corner at $x=0, y=b$ takes the form:

$$
\begin{aligned}
\phi(r, \theta) & \sim \beta_{0}+\beta_{1} r \cos \left[\theta+\chi_{1}\right]+\beta_{2} r^{2} \cos \left[2\left(\theta+\chi_{2}\right)\right]+\beta_{3} r^{2} \cos (2 \theta) \\
& +\beta_{4} r^{4} \cos (4 \theta)+\ldots, \quad r \rightarrow 0
\end{aligned}
$$

where $r=\sqrt{x^{2}+(y-b)^{2}}$ is the distance from this corner and $\chi_{1}, \chi_{2}, \beta_{0}-\beta_{4}$ are arbitrary constants. Again, note that the terms pre-multiplied by $\beta_{1}$ and $\beta_{2}$ arise due to the high order boundary conditions. It is clear from (53) that the fluid velocity and its derivatives are regular in the vicinity of $x=0, y=b$ which indicates that $\hat{E}_{4}=0$. Thus,

$$
w(y)=-E_{3} \psi^{(1)}(y)-\hat{E}_{6} \psi^{(4)}(y)+\alpha^{2} \sum_{n=0}^{\infty} \sum_{q=0}^{\infty} \frac{B_{n} \cosh \left(\gamma_{q} y\right) T_{n q}}{\left(\gamma_{q}^{2}+\mu^{2}\right) D_{q}}, \quad a \leq y \leq b .
$$

It is convenient, henceforth, to use $E_{6}$ instead of $\hat{E}_{6}$.

Having obtained an expression for $w(y)$ in terms of the duct eigenfunctions this is substituted, together with (5) and (6), into the velocity condition (25). On multi- 
plying through by $\alpha \cosh \left(\gamma_{m} y\right)$ and using (9), it is found that

$$
\begin{aligned}
B_{m} & =\frac{\alpha F_{\ell} \eta_{\ell} R_{\ell m}}{s_{m} D_{m}}-\frac{\alpha}{s_{m} D_{m}} \sum_{n=0}^{\infty} A_{n} \eta_{n} R_{n m}-i E_{2} \frac{\gamma_{m} \sinh \left(\gamma_{m} b\right)}{s_{m} D_{m}} \\
& +\frac{i \alpha}{s_{m} D_{m}}\left\{E_{3} \Psi_{m}^{(1)}+E_{6} \Psi_{m}^{(4)}\right\} \\
& -\frac{i \alpha^{3}}{s_{m} D_{m}} \sum_{n=0}^{\infty} B_{n} \sum_{q=0}^{\infty} \frac{T_{q m} T_{q n}}{\left(\gamma_{q}^{2}+\mu^{2}\right) D_{q}}
\end{aligned}
$$

where

$$
\Psi_{m}^{(j)}=\int_{a}^{b} \psi^{(j)}(y) \cosh \left(\gamma_{m} y\right) d y, \quad j=1,4
$$

On using the pressure condition (17) to eliminate the coefficients $A_{n}$, this reduces to

$$
\begin{aligned}
B_{m} & =\frac{2 \alpha F_{\ell} \eta_{\ell} R_{\ell m}}{s_{m} D_{m}}-\frac{\alpha E_{1}}{s_{m} D_{m}} \sum_{n=0}^{\infty} \frac{\eta_{n} \tau_{n} \sinh \left(\tau_{n} a\right) R_{n m}}{C_{n}}-i E_{2} \frac{\gamma_{m} \sinh \left(\gamma_{m} b\right)}{s_{m} D_{m}} \\
& +\frac{i \alpha}{s_{m} D_{m}}\left\{E_{3} \Psi_{m}^{(1)}+E_{6} \Psi_{m}^{(4)}\right\} \\
& -\frac{\alpha^{2}}{s_{m} D_{m}} \sum_{n=0}^{\infty} B_{n} \sum_{q=0}^{\infty}\left\{\frac{\eta_{n} R_{q m} R_{q n}}{C_{q}}+\frac{i \alpha T_{q m} T_{q n}}{\left(\gamma_{q}^{2}+\mu^{2}\right) D_{q}}\right\} .
\end{aligned}
$$

The above equation involves the constants $E_{1}-E_{3}$ and $E_{6}$ which can be found by using (22) together with the edge conditions for the vertical membrane. On recollecting that $E_{1}=0$, and on multiplying $(57)$ by $\gamma_{m} \sinh \left(\gamma_{m} b\right)$, summing over $m$ and using (22), it is found that

$$
\begin{aligned}
E_{2} S_{1}= & -i \alpha \sum_{m=0}^{\infty} \frac{\gamma_{m} \sinh \left(\gamma_{m} b\right)}{s_{m} D_{m}}\left\{2 F_{\ell} \eta_{\ell} R_{\ell m}-E_{1} \sum_{n=0}^{\infty} \frac{\eta_{n} \tau_{n} \sinh \left(\tau_{n} a\right) R_{n m}}{C_{n}}\right\} \\
& +\alpha \sum_{m=0}^{\infty} \frac{\gamma_{m} \sinh \left(\gamma_{m} b\right)}{s_{m} D_{m}}\left\{E_{3} \Psi_{m}^{(1)}+E_{6} \Psi_{m}^{(4)}\right\} \\
& +i \alpha^{2} \sum_{m=0}^{\infty} \frac{\gamma_{m} \sinh \left(\gamma_{m} b\right)}{s_{m} D_{m}} \sum_{n=0}^{\infty} B_{n} \sum_{q=0}^{\infty}\left\{\frac{\eta_{q} R_{q m} R_{q n}}{C_{q}}+\frac{i \alpha T_{q m} T_{q n}}{\left(\gamma_{q}^{2}+\mu^{2}\right) D_{q}}\right\} .
\end{aligned}
$$

It remains to apply appropriate conditions to the edges of the vertical membrane. As mentioned previously, the displacement at $y=a$ is chosen to be zero. That is, $w(a)=0$ which may be expressed as

$$
E_{3} \psi^{(1)}(a)+E_{6} \psi^{(4)}(a)=\alpha^{2} \sum_{n=0}^{\infty} \sum_{q=0}^{\infty} \frac{B_{n} \cosh \left(\gamma_{q} a\right) T_{n q}}{\left(\gamma_{q}^{2}+\mu^{2}\right) D_{q}} .
$$


i) Zero displacement at $y=a$ and $y=b$

Zero displacement at $y=b$ is specified by:

$$
E_{3} \psi^{(1)}(b)+E_{6} \psi^{(4)}(b)=\alpha^{2} \sum_{n=0}^{\infty} \sum_{q=0}^{\infty} \frac{B_{n} \cosh \left(\gamma_{q} b\right) T_{n q}}{\left(\gamma_{q}^{2}+\mu^{2}\right) D_{q}} .
$$

ii) Zero displacement at $y=a$; zero gradient at $y=b$

The zero gradient condition is $w_{y}(b)=0$, that is:

$$
E_{3} \psi_{y}^{(1)}(b)+E_{6} \psi_{y}^{(4)}(b)=\alpha^{2} \sum_{n=0}^{\infty} \sum_{q=0}^{\infty} \frac{B_{n} \gamma_{q} \sinh \left(\gamma_{q} b\right) T_{n q}}{\left(\gamma_{q}^{2}+\mu^{2}\right) D_{q}} .
$$

iii) Zero displacement at $y=a$; impedance at $y=b$

The appropriate condition for the impedance condition, $w_{y}(b)+\xi w(b)=0$, is:

$$
\begin{aligned}
E_{3}\left\{\psi_{y}^{(1)}(b)\right. & \left.+\xi \psi^{(1)}(b)\right\}+E_{6}\left\{\psi_{y}^{(4)}(b)+\xi \psi^{(4)}(b)\right\} \\
& =\alpha^{2} \sum_{n=0}^{\infty} \sum_{q=0}^{\infty} \frac{B_{n} T_{n q}\left\{\gamma_{q} \sinh \left(\gamma_{q} b\right)+\xi \cosh \left(\gamma_{q} b\right)\right\}}{\left(\gamma_{q}^{2}+\mu^{2}\right) D_{q}} .
\end{aligned}
$$

Each set of edge conditions comprises a coupled pair of equations for $E_{3}$ and $E_{6}$. These together with (57) and (58) are truncated and solved numerically.

\section{$5 \quad$ Numerical Results}

The results presented in this section are obtained by truncating the systems of equations obtained in sections 4.1 and 4.2 (that is, (33) for the Galerkin procedure and (57) for the "tailored" approach) and solving the reduced systems together with the appropriate edge conditions. For both the "tailored" approach and the Galerkin method the systems of equations were truncated to 75 terms. The membrane tension and mass are $T=350 \mathrm{~N}$ and $\rho_{m}=0.1715 \mathrm{~kg} \mathrm{~m}^{-2}$. In addition, the fluid parameters are $c=344 \mathrm{~ms}^{-1}$ and $\rho=1.2 \mathrm{~kg} \mathrm{~m}^{-3}$ respectively. The dimensional duct heights are $\bar{a}=0.06 \mathrm{~m}(x<0)$ and $\bar{b}=0.085 \mathrm{~m}(x>0)$.

For the purposes of comparison, figure 2 shows the reflected and transmitted powers for the rigid strip [8] discussed in section 3. Two different incident fields are considered: the structural-borne fundamental mode and the fluid-borne second mode 

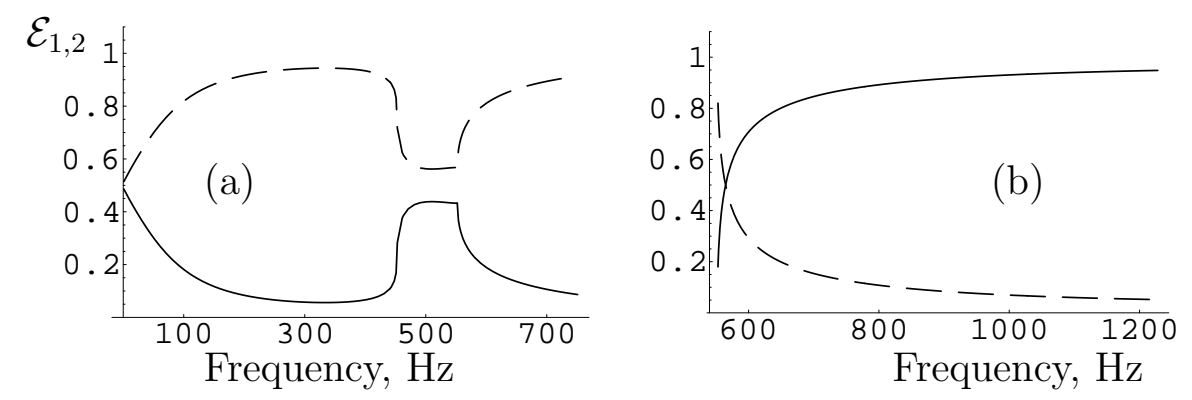

Figure 2: Reflected (dashed) and transmitted (solid) powers against frequency for the Warren et al case (rigid strip), forcing by: (a) fundamental mode $(\ell=0)$ and (b) first higher order mode $(\ell=1)$.

which, for the duct of height $\bar{a}=0.06 \mathrm{~m}$ cuts on at approximately $553 \mathrm{~Hz}$. Note that, for the duct of height $\bar{b}=0.085 \mathrm{~m}$ the first cut-on is at approximately $443 \mathrm{~Hz}$. It is clear from figure 2(a) that the reflected power is significantly reduced (and the transmitted power correspondingly raised) between the two cut-on frequencies.
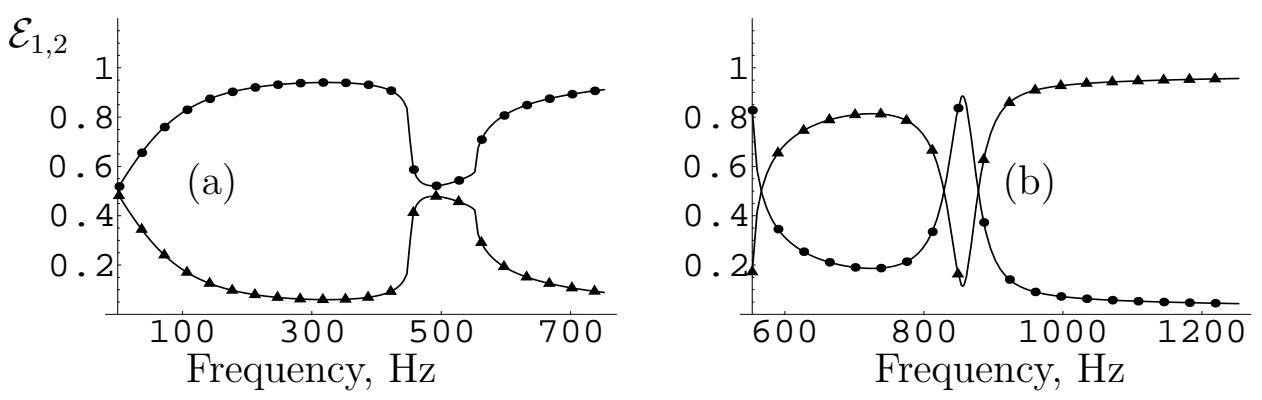

Figure 3: Reflected and transmitted powers against frequency for the membrane strip with $w(a)=w(b)=0$, forcing by: (a) fundamental mode $(\ell=0)$ and (b) first higher order mode $(\ell=1)$.

The results presented in figures 3-5 relate to the membrane strip and comprise a comparison of the reflected and transmitted components of power, plotted against frequency, for each of the three sets of edge conditions discussed in the previous section. As for the case of the rigid strip of figure 2, two different incident fields are considered: the structural-borne fundamental mode and the fluid-borne second mode. In each of figures 3-5 the solid curves are obtained using the "tailored approach" of section 4.2 whilst the symbols ( $\boldsymbol{\Delta}$ for transmitted and $\bullet$ for reflected powers) 
are obtained using the Galerkin method of section 4.1. There is clearly excellent agreement between the methods for all cases considered.

Figure 3 demonstrates that, for the parameter values considered herein, the reflected and transmitted powers do not differ significantly from those for a rigid strip when the vertical membrane displacement is zero at both $y=a$ and $y=b$ and the incident field comprises the fundamental mode. The presence of the bridging membrane becomes apparent, however, when the forcing is due to the first higher order mode: a sharp inversion of the reflected and transmitted powers occurs at approximately 856 $\mathrm{Hz}$.
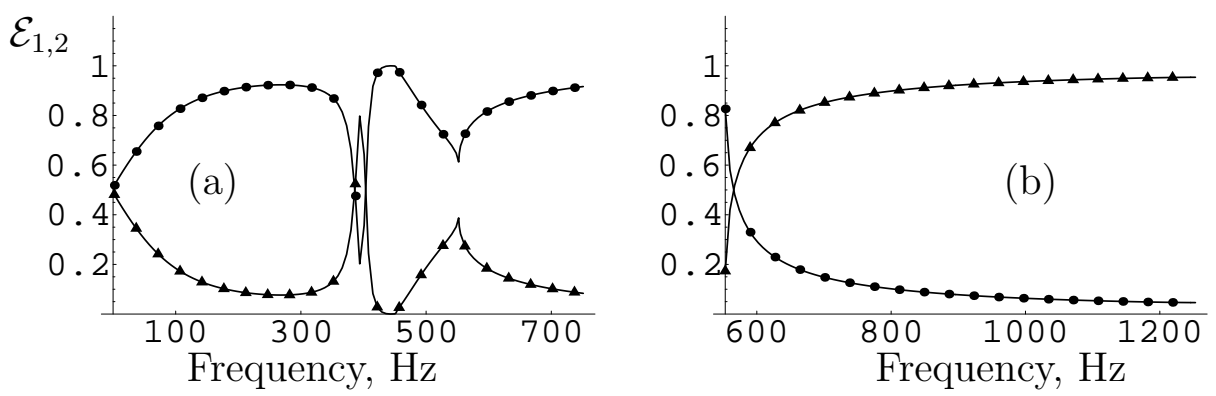

Figure 4: Reflected and transmitted powers against frequency for the membrane strip with $w(a)=w_{y}(b)=0$, forcing by: (a) fundamental mode $(\ell=0)$ and (b) first higher order mode $(\ell=1)$.

In contrast (see figure 4), when the vertical membrane displacement is zero at $y=a$ and the gradient is zero at $y=b$ the reflected and transmitted powers do not differ significantly from those for a rigid strip when the first higher order mode is incident. When the fundamental mode is incident, however, the reflected and transmitted powers differ from those for a rigid strip in that there is an sharp inversion at approximately $437 \mathrm{~Hz}$ and a narrow stop-gap in the frequency range 428-452 Hz (which encompasses the two "cut-ons" mentioned above).

Figure 5 depicts the reflected and transmitted powers when the vertical membrane has zero displacement at $y=a$ and satisfies the impedance or "spring-like" condition $w_{y}(b)+\xi w(b)=0$ at $y=b$ where $\xi=\bar{\xi} / k$ with $\bar{\xi}=15$. Clearly, the condition at $y=b$ reduces to the zero gradient condition as $k$ increases and it is not surprising, 

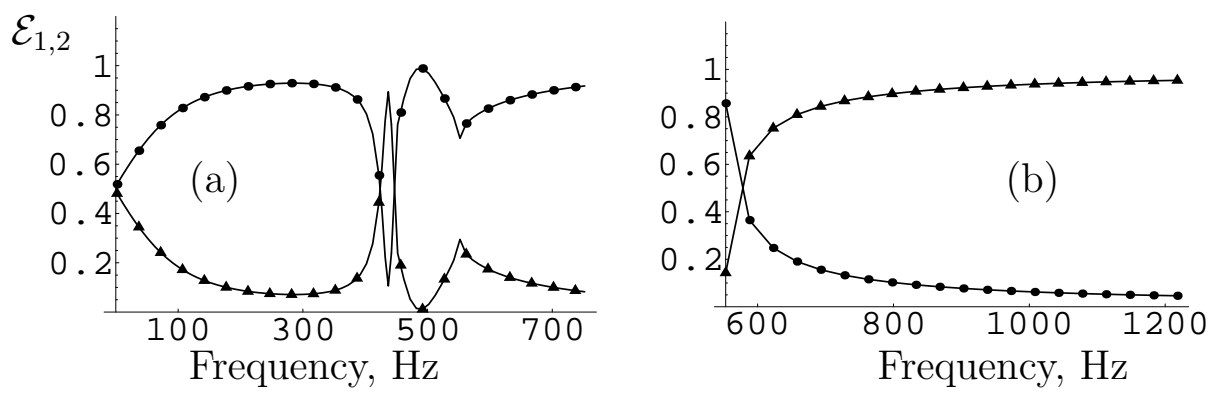

Figure 5: Reflected and transmitted powers against frequency for the membrane strip with $w(a)=w_{y}(b)+\xi w(b)=0, \bar{\xi}=15$, forcing by: (a) fundamental mode $(\ell=0)$ and (b) first higher order mode $(\ell=1)$.

therefore, that figures 4(b) and 5(b) do not differ significantly. Figures 4(a) and 5(a) (fundamental forcing) do show a modest difference.

The results presented in figures 3-5 focus on the reflected and transmitted energies for two different incident waves and three different sets of edge conditions. Bearing in mind (see (52)) that the velocity field is singular in the vicinity of $x=0, y=a$ (that is, $\phi_{\theta} / r=O\left(r^{-1 / 3}\right)$ as $\left.r=\sqrt{x^{2}+(y-a)^{2}} \rightarrow 0\right)$, it is useful to validate the mode-matching method. This can be done by confirming, first, that sufficient terms have been included in the truncation of equations (57) and (58) to ensure that the modal coefficients have converged adequately and, second, that the coefficients contain the correct information to enable the matching conditions, (16) and (25), to be reconstructed. The reader is reminded that Galerkin and the "tailored" approach are, in fact, variations of the same mode-matching approach and thus have very similar convergence properties. Hence, for the purposes of validation, attention is restricted to the "tailored" approach. It is also worth mentioning that a comprehensive study of the convergence of a similar system but with a significantly stronger singularity $\left(\phi_{\theta} / r=O\left(r^{-3 / 4}\right)\right.$ as $\left.r \rightarrow 0\right)$ is presented in [13]. In that article the system was truncated using 80 terms for fundamental mode forcing and 200 terms when the first higher mode was incident. The rate of convergence for such systems is known to be inversely related to the strength of the singularity in the velocity field and, thus, it is expected to achieve good convergence, in both cases, with far fewer terms for the 
problem considered herein.

In fact, the transmitted and reflected energies converge quickly and so the equivalent of figure 6 in [13] (which shows the rate of convergence of the transmitted energy against truncation parameter) is not repeated here. The energy balance is, however, not a good predictor of the level convergence of a system of equations or, indeed, that the solution obtained is in fact correct. It is necessary to confirm that all the matching conditions have been satisfied. Thus, in this section figures showing the real parts of the non-dimensional pressures and velocities at the matching interface are presented for the new "tailored" Galerkin approach. In view of the singularity in the velocity field and the associated Gibb's phenomenon, the velocity fields are computed using the Lanczos filter, of which details are presented in [13]. Thus, for example, instead of plotting simply the truncated form of $\phi_{2 x}(0, y)$ as obtained by differentiating (6) with respect to $x$, the quantity

$$
\phi_{2 x, T}^{\sigma}(0, y)=\sum_{n=0}^{T} B_{n} i s_{n} \sigma_{n} \cosh \left(\gamma_{n} y\right),
$$

is plotted in which

$$
\sigma_{n}=\frac{\sinh \left(\gamma_{n} b / T\right)}{\gamma_{n} b / T}
$$

and $T+1$ is the number of terms to which the system of equations is truncated.

Figure 6 presents (a) the real parts of $\phi_{1}(0, y)$ and $\phi_{2}(0, y), 0 \leq y \leq a$ and (b) the real parts of both sides of equation (25) for the case where the first higher mode is incident $(\ell=1)$ with the zero displacement edge conditions imposed on the bridging membrane at $y=b$. The frequency is $700 \mathrm{~Hz}$ and the system is truncated at $T=74$ (corresponding to 75 terms). Note that $\phi_{1 x}(0, y)$ and $\phi_{2 x}(0, y)$ are computed using the Lanczos filter. The non-dimensional pressures are in excellent agreement and the normal velocities also agree well in the fluid region. Further, as required, $\phi_{2 x}(0, y)=w(y)$ for $a<y \leq b$ where $w(y)$ is given by (49). The corner singularity is apparent in velocity field at at $y=a \sim 0.77$ and it is also clear that there is no singularity at $y=b$. Note that the quantity $w(y)$ satisfies the edge condition $\Re\{w(b)\}=0$ but the fluid velocity is not as accurate at this point. (The reader is advised that the imaginary parts much better satisfy the condition $\Im\{w(b)\}=0$ but 


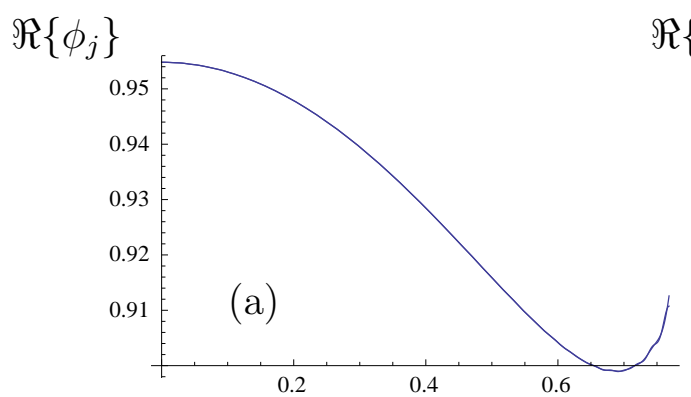

Frequency, $\mathrm{Hz}$

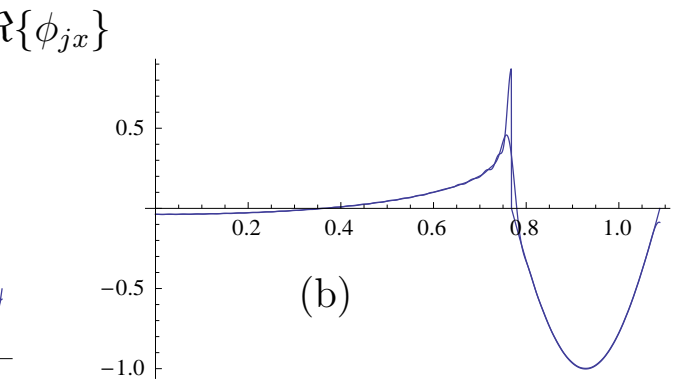

Frequency, $\mathrm{Hz}$

Figure 6: (a) The real parts of $\phi_{1}(0, y)$ and $\phi_{2}(0, y)$, and (b) the real parts of both sides of equation $(25)$ at $700 \mathrm{~Hz}$ for the membrane strip with $w(a)=w(b)=0, \ell=1$.

the real part was selected for presentation to demonstrate the "worst case".)

Figure 7 presents (a) the real parts of $\phi_{1}(0, y)$ and $\phi_{2}(0, y), 0 \leq y \leq a$ and (b) the real parts of both sides of equation (25) for the case where the fundamental mode is incident $(\ell=0)$. The edge conditions imposed on the bridging membrane are zero displacement at $y=a$ and zero gradient at $y=b$. The frequency is $700 \mathrm{~Hz}$ and, as for all previous figures, the system is truncated at $T=74$ (corresponding to 75 terms). Again, the non-dimensional pressures are in excellent agreement, the normal

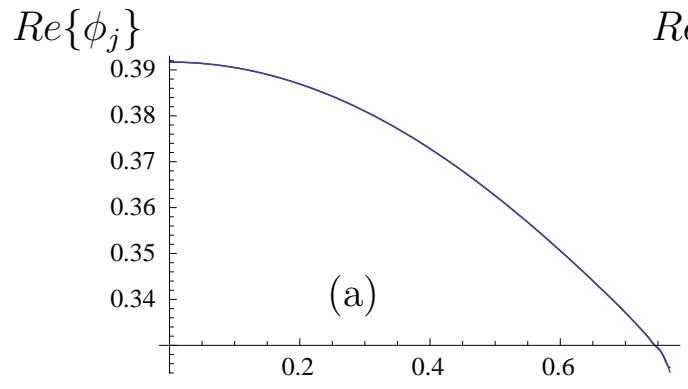

Frequency, $\mathrm{Hz}$

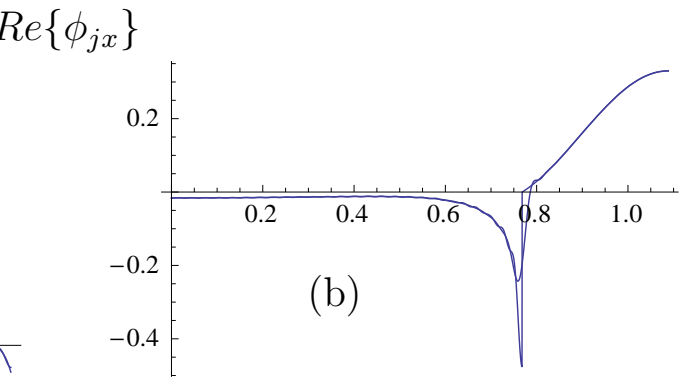

Frequency, $\mathrm{Hz}$

Figure 7: (a) The real parts of $\phi_{1}(0, y)$ and $\phi_{2}(0, y)$, and (b) the real parts of both sides of equation $(25)$ at $700 \mathrm{~Hz}$ for the membrane strip with $w(a)=w_{y}(b)=0$, $\ell=0$.

velocities agree well in the fluid region and $\phi_{2 x}(0, y)=w(y)$ for $a \leq y \leq b$ as required. It is also clear that the edge condition $\operatorname{Re}\left\{w_{y}(b)\right\}=0$ is satisfied. Figures 6 and 7 demonstrate that the matching conditions (16) and (25) have been reconstructed 
using the modal coefficients obtained by truncating equations (57) and (58) together with the appropriate edge conditions. This validates the method and the numerical results presented in figures 3-5.

\section{Discussion}

In this article the scattering of fluid-structure coupled waves in a waveguide with abrupt change in height has been considered. First the problem studied by Warren et al [8] was revisited and then the rigid strip closing the two duct sections was replaced with a membrane. The reflected and transmitted powers have been compared. The results presented in section 5 demonstrate that the conditions applied at the edges of the bridging membrane have a significant effect on the reflected and transmitted powers.

Two solution methods have been used. The first approach is the standard Galerkin procedure in which the displacement is represented as a generalised Fourier series. This method, although conceptually simpler, requires a different set of basis functions for each set of edge conditions applied to the vertical membrane. Further, for some conditions the eigenvalues cannot be expressed explicitly and must be found numerically. The second method (referred to as the "tailored" approach) makes use of the properties of the eigenfunctions for the duct lying in $x>0$ to construct a modal representation for the displacement of the vertical membrane. This approach can deal with a wide range of edge conditions without modification.

As mentioned in the introduction, the problem considered herein is a prototype and that of real interest is the case where each section of membrane is replaced by a thin plate. In this context, it is worth noting that once the vertical membrane is replaced by a thin elastic plate, the eigenvalues for the Galerkin method can be explicitly stated only for the case where both edges are pin-jointed and must be found numerically even for the case where the vertical plate is clamped at one or both ends. Further, there are many practical applications in which the appropriate edge conditions are continuity of, for example, gradient and/or bending moment at the 
junctions between the vertical and horizontal plates. The classical Galerkin method as described in section 4.1 cannot handle such conditions. In contrast the "tailored" approach presented in section 4.2 offers viable means of addressing this class of problem.

\section{Acknowledgments}

MA gratefully acknowledges the financial support of the Higher Education Commission, Pakistan.

\section{References}

[1] Hazard C, Lunéville E (2008) An improved multimodal approach for non-uniform acoustic waveguides. IMA J Appl Math 73:668690.

[2] Kirby R (2008) Modeling sound propagation in acoustic waveguides using a hybrid numerical method. J Acoust Soc Am 124(4):1930-1940.

[3] Kirby R (2009) A comparison between analytic and numerical methods for modelling automotive dissipative silencers with mean flow. J Sound Vib 325:565-582.

[4] Maurel A, Mercier J-F, Pagneux V (2014) Improved mutimodal admittance method in varying cross section waveguides. Proc R Soc A, 470:30130448.

[5] McAlpine A, Wright MCM (2006) Acoustic scattering by a spliced turbofan inlet duct liner at supersonic fan speeds. J Sound Vib 292:911-934 .

[6] Bi W, Pagneux V, Lafarge D, Aurégan Y (2007) An improved multimodal method for sound propagation in nonuniform lined ducts. J Acoust Soc Am 122:280-290.

[7] Lawrie JB, Abrahams ID (1999) An orthogonality condition for a class of problems with high order boundary conditions; applications in sound/structure interaction. Q Jl Mech appl Math 52:161-181.

[8] Warren DP, Lawrie JB, Mohamed IM (2002) Acoustic scattering in wave-guides with discontinuities in height and material property Wave Motion, 36:119-142. 
[9] Lawrie JB (2007) On eigenfunction expansions associated with wave propagation along ducts with wave-bearing boundaries. IMA J. Appl. Math. 72:376-394.

[10] Lawrie JB (2009)Orthogonality relations for fluid-structural waves in a 3-D rectangular duct with flexible walls. Proc R Soc A 465:2347-2367.

[11] Lawrie JB (2012) On acoustic propagation in three-dimensional rectangular ducts with flexible walls and porous linings. J Acoust Soc Am 131(3):1890-1901.

[12] Lawrie JB (2013) Analytic mode-matching for acoustic scattering in three dimensional waveguides with flexible walls: Application to a triangular duct. Wave Motion 50:542-557.

[13] Nawaz R, Lawrie J B (2013) Scattering of a fluid-structure coupled wave at a flanged junction between two flexible waveguides. J Acoust Soc Am 134(3):19391949.

[14] Karmakar D, Bhattacharjee J, Sahoo T (2010) Oblique flexural gravity-wave scattering due to changes in bottom topography. J Eng Math 66:325-341.

[15] Mohapatra SC, Karmakar D, Sahoo T (2011) On capillary gravity-wave motion in two-layer fluids. J Eng Math 71:253-277

[16] Mohapatra SC, Sahoo T (2014) Wave interaction with a floating and submerged elastic plate system. J Eng Math 87:47-71.

[17] Evans DV, Fernyhough M (1995) Edge waves along periodic coastlines. Part 2. J Fluid Mech, 297:307-325.

[18] Gorman D (2000) Free vibration analysis of completely free rectangular plates by the superposition-galerkin method. J Sound Vib 237(5):901-914.

[19] Huang L (2002) Modal analysis of a drumlike silencer. J Acoust Soc Am 112(5):2014-2025.

[20] Huang L(2006) Broadband sound reflection by plates covering side-branch cavities in a duct. J Acoust Soc Am 119(5):2628-2638. 
[21] Liu G, Zhao X, Zhang W and Li S (2014) Study on plate silencer with general boundary conditions. J Sound Vib 333(20):48814896.

[22] Homentcovschi D, Miles RN (2010) Miles A re-expansion method for determining the acoustical impedance and the scattering matrix for the waveguide discontinuity problem. J Acoust Soc Am 128(2):628-638.

[23] Brazier-Smith PR (1987) The acoustic properties of two co-planar half-plane plates. Proc R Soc A 409: 115-139.

[24] Norris AN, Wickham GR (1995) Acoustic diffraction from the junction of two flat plates. Proc R Soc A 451:631-655. 


\section{Figure Legends}

1. The duct geometry (depicting the membrane strip) in which $a=k \bar{a}$ and $b=k \bar{b}$.

2. Reflected (dashed) and transmitted (solid) powers against frequency for the Warren et al case (rigid strip), forcing by: (a) fundamental mode $(\ell=0)$ and (b) first higher order mode $(\ell=1)$.

3. Reflected and transmitted powers against frequency for the membrane strip with $w(a)=w(b)=0$, forcing by: (a) fundamental mode $(\ell=0)$ and (b) first higher order mode $(\ell=1)$.

4. Reflected and transmitted powers against frequency for the membrane strip with $w(a)=w_{y}(b)=0$, forcing by: (a) fundamental mode $(\ell=0)$ and (b) first higher order mode $(\ell=1)$.

5. Reflected and transmitted powers against frequency for the membrane strip with $w(a)=w_{y}(b)+\xi w(b)=0, \bar{\xi}=15$, forcing by: (a) fundamental mode $(\ell=0)$ and (b) first higher order mode $(\ell=1)$.

6. (a) The real parts of $\phi_{1}(0, y)$ and $\phi_{2}(0, y)$, and (b) the real parts of both sides of equation (25) at $700 \mathrm{~Hz}$ for the membrane strip with $w(a)=w(b)=0, \ell=1$.

7. (a) The real parts of $\phi_{1}(0, y)$ and $\phi_{2}(0, y)$, and (b) the real parts of both sides of equation $(25)$ at $700 \mathrm{~Hz}$ for the membrane strip with $w(a)=w_{y}(b)=0, \ell=0$. 